\title{
Joint uplink and downlink capacity considerations in admission control in multiservice CDMA/HSDPA systems
}

\author{
Eitan Altman \\ INRIA-Sophia Antipolis \\ 2004 route des Lucioles - BP \\ 93 \\ 06902 Sophia Antipolis, \\ France \\ eitan.altman@sophia.inria.fr \\ Tijani Chahed \\ GET/INT - UMR CNRS 5157 \\ 9 rue $C$. Fourier \\ 91011 Evry CEDEX, France \\ tijani.chahed@int-edu.eu
}

\author{
Salah-Eddine Elayoubi \\ France Telecom R\&D \\ 38, rue du Général Leclerc \\ 92794 Issy les Moulieaux, \\ France \\ salaheddine.elayoubi@orange- \\ ftgroup.com
}

\begin{abstract}
TCP-based data flows generate packets and ACKs in two directions, be it in the wireline or wireless networks. In the latter case, packets are typically found in the downlink whereas ACKs are in the uplink. Those two links are asymmetric in the case of CDMA-based High Data Rate (HDR)/High-Speed Downlink Packet Access (HSDPA) systems, the uplink being much slower than the downlink and thus, in some cases, restrictive in terms of the achievable throughput of the TCP flow. The aim of this work is to evaluate the performance of such a setting, in the presence of both streaming and elastic traffic, under a dynamic scenario where users arrive to the system and leave it after completion of their service. We specifically quantify the impact of the uplink on the overall performance of TCP and study the model variations according to several parameters such as load, file size and radio conditions ${ }^{1}$.
\end{abstract}

\section{Keywords}

Capacity, joint uplink and downlink, HSDPA/HDR, TCP

\section{INTRODUCTION}

The choice that has been made in CDMA-based systems to accompany the arrival of data-oriented applications in the third generation mobile systems is to offer larger bit rates in the downlink where most of these applications are expected to be. And so the overall system became asymmetric, with lower rate uplink, mostly based on Dedicated CHannels (DCHs), suitable for streaming applications such as voice, and higher bit rate downlink, implementing High Data Rate (HDR) [1]/High-Speed Downlink Packet Access (HSDPA) [2], and which offers packet-based applications a high-rate shared medium. ${ }^{1}$ This work was jointly supported by INRIA ARC IFANY
and Euro-NGI/FGI specific project CELLULAR

Permission to make digital or hard copies of all or part of this work for personal or classroom use is granted without fee provided that copies are not made or distributed for profit or commercial advantage and that copies bear this notice and the full citation on the first page. To copy otherwise, to republish, to post on servers or to redistribute to lists, requires prior specific permission and/or a fee.

Valuetools '07, October 23-25, 2007, Nantes, France

Copyright 2007 ICST 978-963-9799-00-4.
Elastic flows however typically use TCP in the transport layer, with packets flowing on the higher-rate downlink, with instantaneous rates up to some $10 \mathrm{Mbps}$, the ACKs, as a flow on their own, returning on the slower rate uplink, with rates which can be as small as $16 \mathrm{Kbps}$. The asymmetry in architecture may result in cases where the uplink is restrictive and does not let data flows take full advantage of all the available capacity in the downlink.

So far, to the best of our knowledge, works in CDMA systems considered capacity separately in the uplink [3] and downlink [4]. The only work that we are aware of that considers the joint capacity in the uplink and downlink is contained in Reference [5]. It however considers dedicated channels only and not HSDPA and the data flows are considered in one direction only, i.e., it does not take into account the flow of ACKs in the opposite direction.

Other works considered asymmetry in links and quantified their impact on TCP performance (for instance Reference [6] and references cited therein). These works however consider solely the wireline context, are mostly carried out at the packet level and do not consider a mixture of streaming and elastic flows.

In this work, we investigate the simultaneous joint capacity of the uplink and downlink in the presence of both streaming and elastic flows, the latter governed by TCP at the transport layer, with packets on the downlink and ACKs returning back in the uplink. The main difference between those types of flows is that streaming flows require some constant bit rate but their service duration is independent of the quantity of resources they get. This is not the case of data flows which first have the ability to share resources in a fair manner among themselves and second would leave the system sooner if they get more resources.

Please note that the case of packets in the uplink and corresponding ACKs in the downlink is less typical and less restrictive with respect to our problem statement; our model and analysis are however applicable to that case and to the case with packets and ACKs in both links too.

The remainder of this work is organized as follows. In Section II, we show our model for both the uplink and downlink and detail the arrival and departure processes and mean rates of both streaming and elastic flows. In the next section we show our analysis based on the Quasi-Birth Death (QBD) process with a matrix-geometric solution to the steady-state probabilities of the system. In Section IV, we show some performance evaluation results to illustrate the impact of the 
uplink on the overall TCP performance and this with respect to several system parameters, namely load, file size and radio conditions. Section V contains some analysis on how to make the uplink less restrictive by means of squeezing the streaming flows present in the uplink. Section VI eventually concludes the paper. In the appendix, we develop analytical models for the capacity and throughputs in UMTS/HSDPA systems, that we use in our modeling and analysis hereafter.

\section{MODEL}

Let the uplink be modeled as a server with capacity $C_{u}$ and let the downlink be modeled as a server with capacity $C_{d}$. The term capacity refers to the net capacity, in units of bandwidth or normalized to units of flows, and takes into account all the physics of the radio interface, such as interference and power. For the sake of readability of this paper, we leave the derivation of these capacity values to the appendix.

Let the arrival of streaming flows be Poisson with mean arrival rate $\lambda_{u}^{s}$ in the uplink and $\lambda_{d}^{s}$ in the downlink. These flows are assumed to have a service exponentially distributed with mean duration equal to $T^{s}$ or equivalently a mean service rate $\mu^{s}=1 / T^{s}$. Each streaming flow is transported over a dedicated link with rate $R_{u}^{s}$, for instance $16 \mathrm{Kbps}$. Again, the service duration is independent of the amount of capacity granted to this type of flow.

We consider for the time being that those two sets of streaming flows, uplink and downlink, are independent and that the maximum number of such flows in the uplink is $N_{u}$ and in the downlink $N_{d} ; N_{u} \leq C_{u}$ and $N_{d} \leq C_{d}$. If we are to model interactive streaming traffic, such as telephony, the number of flows of this type of traffic should be the same in both directions.

Let $\lambda_{d}^{d}$ denote the arrival rate for data flows in the downlink. We adopt an admission scheme that gives priority to voice flows over data ones. Based on this, data flows share (fairly) the available capacity left over by streaming ones. Once in the system, we assume that this data packet flow generates instantaneously a corresponding stream of ACKs in the uplink. Now this overall data flow, packets and ACKs, shall take the minimum capacity between the bandwidth left over by streaming flows in the downlink to process data packets and the bandwidth left over by streaming flows in the uplink to process smaller size ACKs. Note that we assume that ACKs are transported over shared channels in the uplink too.

And so, in total, each data flow obtains on average, some $R^{d}$ throughput given by:

$$
R^{d}=\min \left(\frac{T_{d}^{d}\left(X_{d}^{s}(t)\right)}{X^{d}(t)}, \frac{T_{u}^{d}\left(X_{u}^{s}(t)\right)}{X^{d}(t)} \frac{s_{p} b}{s_{a}}\right)
$$

where $X^{d}$ is the number of concurrent data flows in the system, $X_{u}^{s}$ is the number of streaming flows in the uplink, $X_{d}^{s}$ is the number of streaming flows in the downlink, $b$ is the number of packets acknowledged by a cumulative ACK, $s_{p}$ is the packet size and $s_{a}$ is the ACK size. $T_{u}^{d}$ and $T_{d}^{d}$ are the throughputs achieved by data calls in the uplink and downlink, respectively, and are calculated in the appendix (Eqns. (14) and (19)).

Remark 1. In the above, we assumed that data flows are managed through some round robin scheduling algorithm in the downlink. It is however common to use some kind of opportunistic scheduling in HSDPA-based systems, such as Proportional Fair Scheduling (PFS). In this case, a gain function representing the radio conditions of a given user [9] must be added to the throughput shown in the appendix.

We assume that streaming flows are subject to admission control. The CAC ensures in this case that the capacities in both uplink and downlink are not exceeded, i.e., $X_{i}^{s} \leq N_{i}$, $i=1,2$. This is not the case of elastic flows which are served on a shared basis and to which we do not apply an admission control.

Remark 2. It should be clear that the resources used by data flows are function of their number as well as the number of streaming calls in progress in the system, i.e., we should have written $R^{d}(X)$ where $X$ is a vector denoting the number of streaming and elastic flows $X^{s}$ and $X^{d}$ respectively. As will be seen in the last section of this paper, even $R^{u}$, the throughput of streaming flows, will be made dependent on $X$. We however drop $(X)$ for the sake of notational convenience.

\section{ANALYSIS}

Now, our system of asymmetric uplink and downlink transporting streaming and elastic flows modeled above can be solved as follows [8]. The number of streaming flows in progress $X_{i}^{s}(t), i=u, d$, is a birth-death process with parameters $\lambda_{i}^{s}$ and $\mu^{s}$. The steady state probabilities $\pi($.$) are$ given by the Erlang formula as:

$$
\pi\left(X_{i}^{s}=x\right)=\frac{1}{\sum_{k=0}^{N_{i}} \frac{\left(\rho_{i}^{s}\right)^{k}}{k !}} \frac{\left(\rho_{i}^{s}\right)^{x}}{x !}
$$

where $\rho_{i}^{s}=\lambda_{i}^{s} / \mu^{s}$

The blocking probability $B_{i}^{s}, i=u, d$, of streaming flows is given by:

$$
B_{i}^{s}=\frac{1}{\sum_{k=0}^{N_{i}} \frac{\left(\rho_{i}^{s}\right)^{k}}{k !}} \frac{\left(\rho_{i}^{s}\right)^{N_{i}}}{N_{i} !}
$$

The process $\left(X_{u}^{s}(t), X_{d}^{s}(t), X^{d}(t)\right)$ referring to the number of streaming flows in the uplink and downlink as well as the number of data flows, respectively, is a homogeneous QuasiBirth and Death (QBD) process with infinitesimal generator $Q$ given by:

$$
\mathbf{Q}=\left[\begin{array}{ccccc}
B & A_{0} & 0 & 0 & \ldots \\
A_{2} & A_{1} & A_{0} & 0 & \ldots \\
0 & A_{2} & A_{1} & A_{0} & \ldots \\
. & . & . & . & \ldots \\
. & . & . & . & \ldots
\end{array}\right]
$$

where $B, A_{0}, A_{1}$ and $A_{2}$ are square matrices of size $\left(N_{u}+\right.$ 1) $\left(N_{d}+1\right)$ which we denote by $N$ (Recall that $N_{u}$ and $N_{d}$ are the maximum number of streaming flows that are admitted to the system in the uplink and downlink respectively). $A_{0}$ represents the data flows arrivals, with arrival rates at the diagonal, $A_{2}$ represents their departures, with mean departure rates, given by the HSDPA scheduling and divided by the mean file size, at the diagonal too. $A_{1}$ corresponds to the arrival and departure of voice flows. It is tri-diagonal, with mean arrival rates at the upper diagonal and departure rates at the lower one. The diagonal entries are simply the 
negative sum of all other entries at the same row, which are arrival and departure rates of voice and data flows, so as to make the sum of the elements of the row of $Q$ equal to zero. Eventually, $B=A_{1}+A_{2}$.

The steady-state equations are given by:

$$
\pi(0) B+\pi(1) A_{2}=0
$$

and

$$
\pi(i-1) A_{0}+\pi(1) A_{1}+\pi(i+1) A_{2}=0 ; i \geq 0
$$

Following a matrix-algebraic approach, we can solve this QBD process as follows [10].

If the system is stable, the steady-state probabilities are given by:

$$
\pi(i)=\pi(0) S^{i}
$$

where $S$ is a minimal non-negative matrix solution to the equation:

$$
A_{0}+S A_{1}+S^{2} A_{2}
$$

and can be solved recursively as follows:

$$
S=\left(A_{0}+S T+S^{2} A_{2}\right) D^{-1}
$$

starting from $S=0$.

Matrices $T$ and $D$ are such that $A_{1}=T-D, T$ having zero diagonal and $D$ a diagonal matrix, positive and invertible.

Now, $\pi(0)$ can be obtained using Eqn. (4) and the normalization equation $\pi e=1$ which is in this case equivalent to $\pi(0)(I-S)^{-1} e=1$.

The number of data calls have thus the following marginal probability:

$$
\operatorname{Pr}\left(X^{d}=i\right)=\sum_{j=0}^{N} \pi(i, j)=\pi(i) e=\pi(0) S^{i} e
$$

Now, to compute the above equation, let matrix $V$ be a diagonal matrix containing the eigenvalues of $S, s_{i}$, and let $W$ be a matrix containing its eigenvectors, $w_{i}$, as columns. We can thus re-write the above equation as [7]:

$$
\operatorname{Pr}\left(X^{d}=i\right)=\pi(0) W V^{i} W^{-1} e=\sum_{j=0}^{N} a_{j} s_{j}^{i}
$$

where $a_{j}=\pi(0) w_{j} e_{j}^{\prime} W^{-1} ; e_{j}^{\prime}$ being a zero vector of proper dimension with 1 at the $j$ th entry.

Eventually, the mean number of data flows is given by:

$$
\bar{N}^{d}=\sum_{j=0}^{N} a_{j} \frac{s_{j}}{\left(1-s_{j}\right)^{2}}
$$

and the mean transfer time (Little):

$$
T^{d}=\bar{N}^{d} / \lambda^{d}
$$

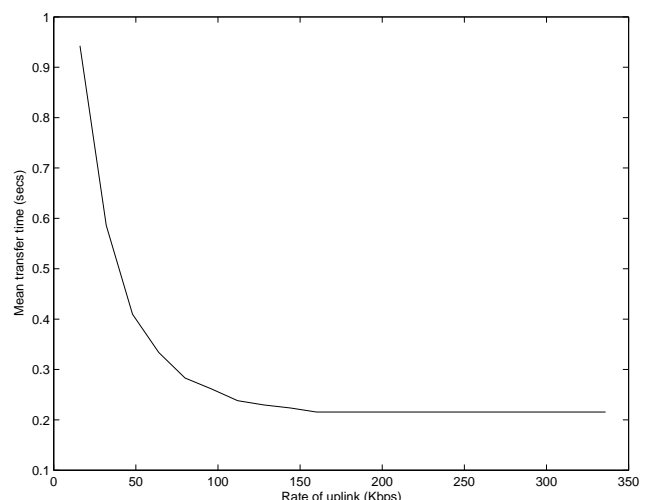

Figure 1: Mean transfer time of data flows

We now evaluate numerically the performance of our system. In the following, let the capacity of the dedicated channels be equal to $R_{u}^{s}$ bandwidth and which we vary starting from $16 \mathrm{Kbps}$. Let $C_{d}$, the capacity of the downlink, be equal to $10 \mathrm{Mbps}$. Let the thresholds on the number of streaming flows in the uplink and downlink $N_{u}=N_{d}=5$.

Unless otherwise stated, let us consider that users experience the same radio conditions on average and are at some $200 \mathrm{~m}$ distance from the base station (refer to Figure 7 ). We take also a file size equal to 100 packets of size 1500 bytes each. Eventually, the mean arrival rates for streaming flows in both the uplink and downlink $\lambda_{i}^{s}, i=u, d$, are taken as 0.2 flows/sec, the mean service rate $\mu^{s}=0.1$ and the mean arrival rate for elastic flows equal to 0.5 flows/sec.

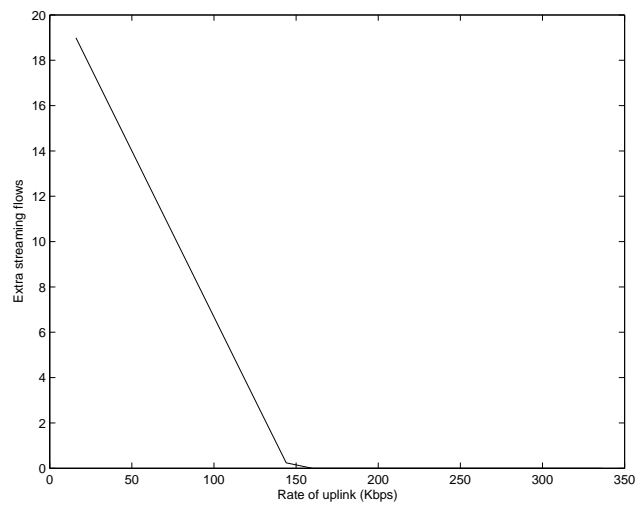

Figure 2: Extra streaming flows

\subsection{Impact of uplink}

Figure 1 shows the mean transfer time of a TCP file for different values of the uplink rate. We observe that below a certain value, $160 \mathrm{Kbps}$ in this case, the uplink is restrictive (and should thus be taken into account into acceptance constraints). Beyond that value, it is not.

\section{PERFORMANCE EVALUATION}




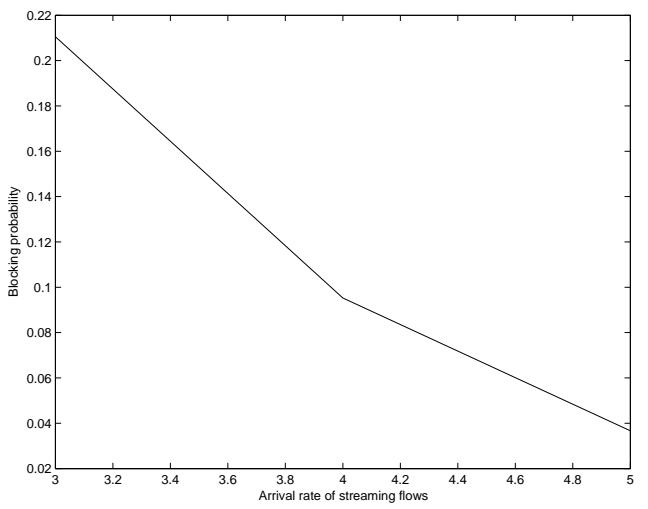

Figure 3: Blocking rates for streaming flows

Now, each time that the uplink is restrictive to data flows, i.e., below $160 \mathrm{Kbps}$, some extra streaming flows can be accommodated in the downlink. Their number as a function of different values of the uplink rate is shown in Figure 2.

\subsection{Impact of load}

We now change the upper bound on the number of streaming flows in the system, from 3 to 5 flows per second, and show in Figures 3 and 4 the blocking rate of streaming flows as well as the mean transfer time of data ones.

Naturally, the blocking probability decreases as the threshold increases. This blocking results in a larger number of streaming flows in progress in the system and hence a worse performance, in terms of lower mean transfer time, for elastic ones.

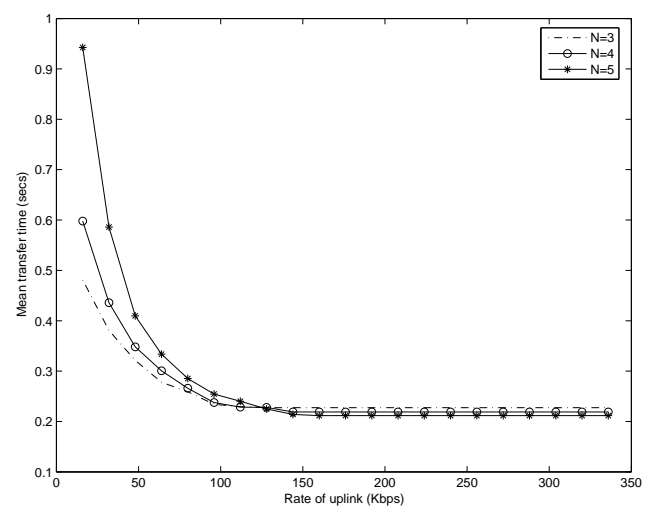

Figure 4: Mean transfer time of data flows

For a fixer threshold on streaming flows (equal to 5), changing the load of elastic flows by changing the mean arrival rate from 0.2 to 2.0 flows/sec yields to the mean transfer performance contained in Figure 5 and which increases as the load of elastic flows increases.

\subsection{Impact of file size}

We now change the file size, from 10 to 100 packets, and

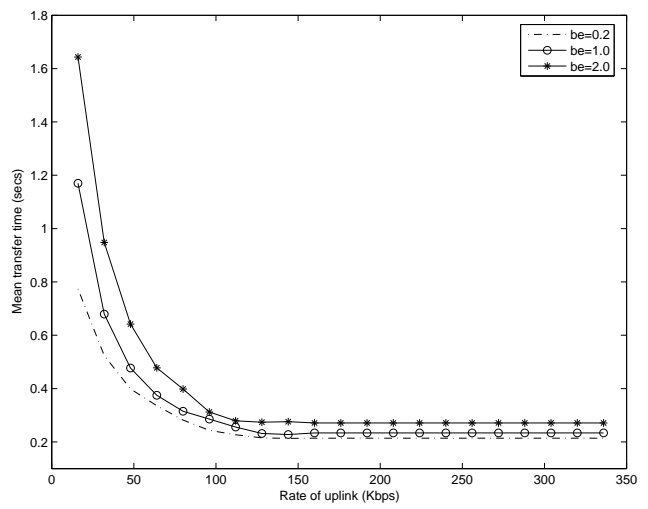

Figure 5: Mean transfer time of data flows

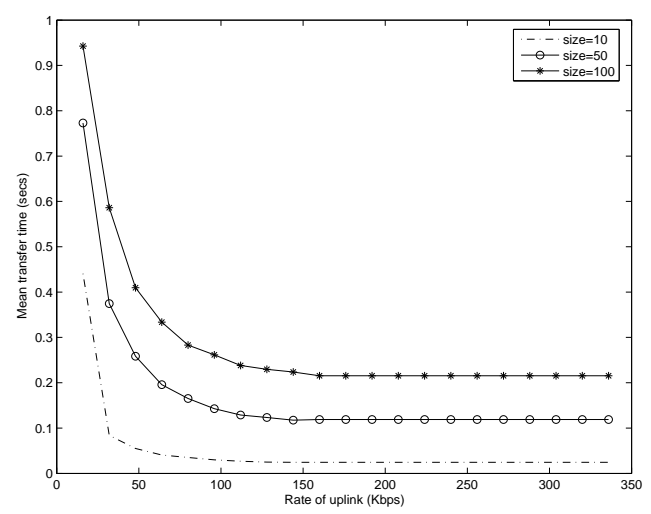

Figure 6: Mean transfer time of data flows

plot in Figure 6 the corresponding mean transfer time for different values of the uplink. The mean performance of TCP flows degrades as the file to be transferred gets larger.

\subsection{Impact of radio conditions}

We now see how the uplink is restrictive in terms of radio conditions. We change the latter from good to bad by increasing the distance from 100 to 200 and $250 \mathrm{~m}$. Indeed, as can be seen from Eqn. (14), the throughput achieved for HSDPA depends on the position in the cell. This is illustrated in Figure 7, where we plot the throughput achieved for a cell that contains only HSDPA users (i.e. emits on the DSCH channel with maximal power), function of the position in the cell.

We show in Figure 8 the corresponding mean transfer time for different values of the uplink rate. We observe that when the uplink is most restrictive, all types of users are penalized in the same way. The discrepancy between users changes as the uplink becomes less restrictive, and in this case, the radio conditions play a role too, i.e., users far from the base station have a degraded performance not because of a lesser available capacity but because they cannot take full advantage of it. 


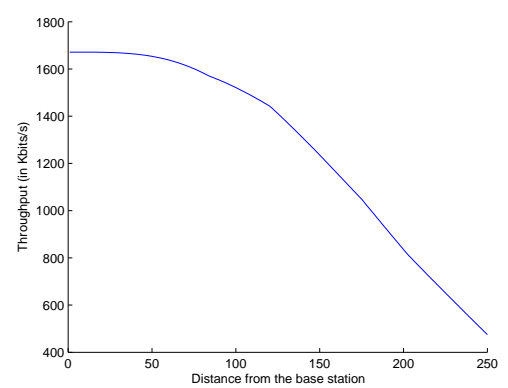

Figure 7: Achieved throughput as a function of the distance

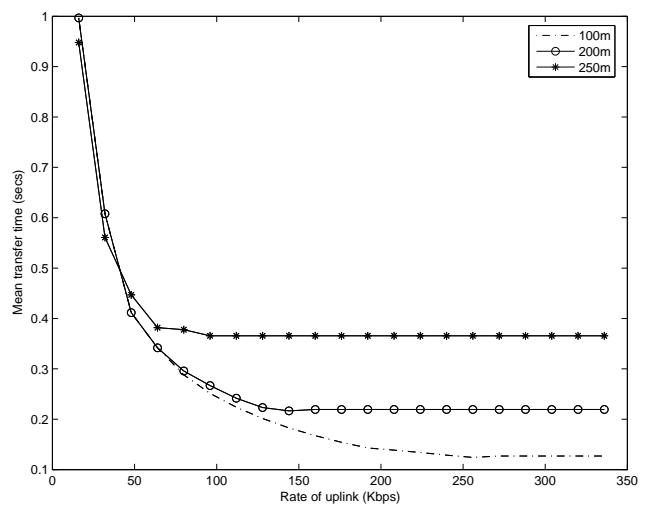

Figure 8: Mean transfer time of data flows

\subsection{Impact of cumulative ACK}

We eventually show how the uplink is restrictive in terms of different values of cumulative ACK, namely $b=1$ and 2. Figure 9 shows the corresponding mean transfer time for different values of the the uplink and we can observe that a larger value of $b$ limits the problem of the uplink but does not solve it.

\section{EXTENDING THE ERLANG CAPACITY REGION}

The previous section illustrated the regions where the uplink is restrictive and how this restriction changes with load, file size and radio conditions. The question we pose now is how to remedy to such a situation. One answer goes as follows. Whenever the uplink is restrictive, we can actually take advantage of the fact that CDMA uses AMR codec for streaming applications and which allows for eight different transmission rates $R_{u, i}^{s}, i=1, \ldots 8[11]\left(R_{u, j}^{s}>R_{u, k}^{s}\right.$ for $j<k$ ). In this case, we can squeeze the rate of transmission of each streaming flow in the uplink and make hence more room for ACKs to accompany the higher rate of packets in the downlink and make thus the uplink less restrictive.

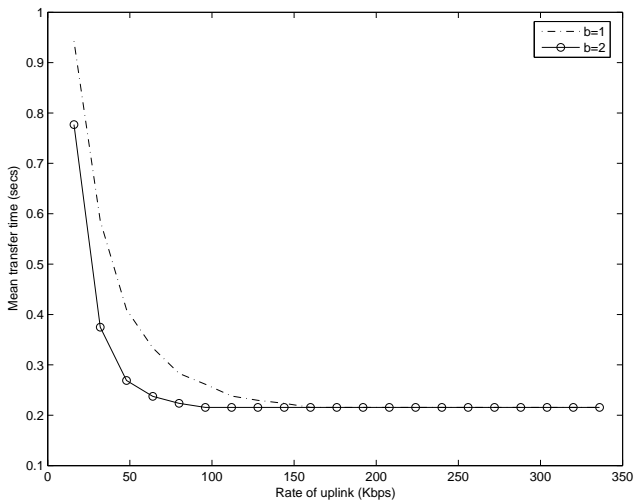

Figure 9: Mean transfer time of data flows

The need for squeezing is obtained whenever the capacity left over by streaming flows in the uplink is less than that of the downlink, or:

$$
\frac{T_{d}^{d}\left(X_{d}^{s}(t)\right)}{X^{d}(t)}>\frac{T_{u}^{d}\left(X_{u}^{s}(t)\right)}{X^{d}(t)} \frac{s_{p} b}{s_{a}}
$$

where, again, $X^{d}$ is the number of concurrent data flows in the system, $X_{u}^{s}$ is the number of voice flows in the uplink, $X_{d}^{s}$ is the number of data flows in the downlink, $b$ is the number of packets acknowledged by a cumulative ACK, $s_{p}$ is the packet size, $s_{a}$ is the ACK size and $T_{u}^{d}$ and $T_{u}^{d}$ are the throughputs achieved by data calls in the uplink and downlink, respectively.

The difference between the two terms for uplink and downlink capacities mentioned in Eqn. (9), hereby denoted by $\delta$, indicates how much capacity is not being used and consequently how much squeezing is needed. This translates evenly among on-going streaming calls which will now have a rate $R_{u}^{s}(x)$ equal to $R_{u}^{s}-\delta / X_{u}^{s}$ rounded up to the nearest value of $R_{u, i}^{s}, i=2, \ldots 8$.

The overall utility $E[R]$ perceived by streaming flows undergoing both phases, squeezed and not squeezed, is the mean of the individual utility of each phase. And is given by [7]:

$$
E[R]=\frac{\sum_{i=1}^{N_{u}} \operatorname{Pr}\left(X_{u}^{s}(t)=x\right) x R_{u}^{s}(x)}{\sum_{i=1}^{N_{u}} \operatorname{Pr}\left(X_{u}^{s}(t)=x\right) x}
$$

The analysis follows the same QBD process as above.

Remark3. Please note that it is not possible to squeeze streaming flows so as to accommodate other streaming flows. Indeed, squeezing degrades performance and is only acceptable for short periods of times, such as above, to make more room for short-sized ACKs. Long periods of squeezing, which will result in the case of admitting other streaming flows, causes the dropping of the squeezed streaming flows.

\section{CONCLUDING REMARKS}

We investigated in this work the joint capacity of the uplink and downlink in CDMA-based system where both directions of flows are asymmetric due to the implementation of HDR/HSDPA in the downlink which makes it capable to 
offer higher bit rates than the classical uplink. The notable impact of such an asymmetry is on the transport of TCPbased data flows which use ACKs, typically in the uplink, whereas data packets enjoy higher bit rates in the downlink. In this case, the uplink may turn out to be restrictive. This restriction is exacerbated by higher load, larger file size and worst radio conditions.

In this work, we considered that ACKs are carried over shared links in the uplink. The case of dedicated links for ACKs is a little bit different. In this case, the maximum number of elastic flows that can be admitted to the system is limited by the uplink, as the latter imposes a lower limit on the resources given to each flow of ACKs. This is not the case of the downlink where data flows can share the leftover capacity with no lower bound on the resulting individual share. The analysis would follow a Level-Dependent QBD (LDQBD) process and can be carried out using numerical methods similar to the ones used in this paper [7].

In this case, one interesting way to extend the capacity of the system beyond its limits is to suppress ACKs. This is possible if one adopts an open-loop reliable transport, such as the one based on Luby-Transform (LT) codes [12]. This is an issue of future work perspective.

\section{REFERENCES}

[1] P. Bender, P. Black, M. Grob, R. Padovani, N. Sindhushayana, A. Viterbi, CDMA/HDR: A Bandwidth-Efficient High-Speed Wireless Data Service for Nomadic Users, IEEE Communications Magazine, July 2000 .

[2] T. Chahed, M. Dirani, Cross-layer modeling of capacity in UMTS/HSDPA networks under dynamic user setting, VTC'2006-Fall, Montreal, September 2006.

[3] E. Altman, Capacity of multi-service cellular networks with transmission-rate control: : a queueing analysis, ACM Mobicom'02, Atlanta, September 2002.

[4] S-E. Elayoubi, T. Chahed, G. Hébuterne, Mobility-aware admission control schemes in the downlink of third generation wireless systems, IEEE Transactions on Vehicular Technology, January 2007.

[5] W. Jeon and D. Jeong, Call Admission Control for Mobile Multimedia Communications with Traffic Asymmetry between Uplink and Downlink, IEEE Transactions on Vehicular Technology, Volume : 50 Issue: 1 , January 2001.

[6] T. V. Lakshman, U. Madhow, B. Suter, TCP/IP performance with random loss and bidirectional congestion. IEEE/ACM Trans. Netw. 8(5): 541-555, 2000.

[7] N. Hegde, E. Altman, Capacity of Multiservice WCDMA Networks with Variable GoS, Wireless Networks, 12(2), April 2006.

[8] G. Alpan, E. Altman, H. Magroun, D. Kofman, Call admission control in the presence of point-to-multipoint best-effort connections, ICC'99.

[9] T. Bonald, A. Proutiere, Wireless downlink data channels : User performance and cell dimensionning, ACM Mobicom, 2003.

[10] M. F. Neuts, Matrix-Geometric Solutions in Stochastic Models, an Algorithmic Approach, Dover publications, 1994
[11] H. Holma, A. Toskala, WCDMA for UMTS, John Wiley and Sons Ltd., 2001.

[12] M. Luby, LT codes, IEEE-FOCS, pp. 271-282, 2002.

[13] K. Sipila, Z. Honkasalo, J. Laiho-Steffens and A. Wackr, Estimation of capacity and required transmission power of WCDMA downlink based on a downlink pole equation, IEEE VTC 2000.

[14] P. Bender, P. Black, M. Grob, R. Padovani, N. Sindhushayana, A. Viterbi, CDMA/HDR: A bandwidth-efficient high-speed wireless data service for nomadic users, IEEE Communications Magazine, July 2000.

\section{APPENDIX}

\section{A. DOWNLINK MODEL}

In the downlink, if cell 0 contains $X_{d}$ active users, the Signal-to-Interference plus Noise Ratio (SINR) achieved for user $k$, situated at distance $d_{k}$ from its own base station, is given by:

$$
S I N R_{k}=\frac{P_{k, 0} / q_{k, 0}}{I_{\text {inter }, k}+I_{\text {intra }, k}+N_{0}} S_{k}
$$

where $P_{k, 0}$ is the power used by the base station of cell 0 towards user $k, q_{k, 0}$ is the path loss between the target base station and user $k$ and which depends on the distance $d_{k}$, $I$ is the interference (both intra- and inter-cell), $N_{0}$ is the noise term and $S_{k}$ is the spreading factor.

To analyze the interference, let us first note that the intracell interference originates from the common channels and from other users: $I_{\text {intra }}\left(d_{0}\right)=\alpha\left(P_{t o t, 0}-P_{k, 0}\right) / q_{k, 0}, \alpha$ being the orthogonality factor and $P_{t o t, l}$ the total transmitted power by base station $l$. For the inter-cell interference, it is given by: $I_{\text {inter }, k}=\sum_{l \neq 0} P_{t o t, l} / q_{k, l} . P_{\text {tot }, l}=\chi P_{\max }$, where $\chi$ is the average load in a typical cell of the system, defined as the ratio between the used and total powers.

On the other hand, the value $\sum_{l \neq 0} q_{k, 0} / q_{k, l}$ is the wellknown F-factor $F_{k}$ [11] [13]. The SINR is then equal to

$$
S I N R_{k}=\frac{P_{k, 0}}{\alpha\left(P_{t o t, 0}-P_{k, 0}\right)+\chi P_{\max } F_{k}+N_{0} q_{k, 0}} S_{k}
$$

leading to the expression:

$$
\beta_{k}=\frac{P_{k, 0}}{\alpha P_{t o t, 0}+\chi P_{\max } F_{k}+N_{0} q_{k, 0}}
$$

where we define

$$
\beta_{k}=\frac{S I N R_{k}}{S_{k}+\alpha \cdot S I N R_{k}}
$$

If $X_{d}^{s}$ streaming users, with no HSDPA users, were present in the cell, this leads to the power expression:

$$
P_{t o t, 0}=\frac{P_{C C H}+\beta_{v} \sum_{k}\left(\chi P_{\max } F_{k}+N_{0} q_{k, 0}\right)}{1-\alpha X_{d}^{s} \beta^{s}}
$$

where $\beta^{s}=\frac{S I N R^{s}}{S+\alpha . S I N R^{s}}, P_{C C H}$ is the power associated with the Common CHannels (CCHs).

Considering the average values over the cell, we have: 


$$
P_{t o t, 0}=\frac{P_{C C H}+\beta^{s}\left(\chi P_{\max } \bar{F}+N_{0} \bar{q}\right) X_{d}^{s}}{1-\alpha \beta^{s} X_{d}^{s}}
$$

Considering the constraint on the maximal transmission power $\left(P_{t o t, 0} \leq P_{\max }\right)$, the constraint on the number of users becomes:

$$
\beta^{s}\left(\chi P_{\max } \bar{F}+N_{0} \bar{q}+\alpha P_{\max }\right) X_{d}^{s} \leq P_{\max }-P_{C C H}
$$

The capacity of the system in the downlink $C_{d}$ is equal to:

$$
C_{d}=\frac{P_{\max }-P_{C C H}}{\beta^{s}\left(\chi P_{\max } \bar{F}+N_{0} \bar{q}+\alpha P_{\max }\right)}
$$

However, if the number of HSDPA calls is nonzero, the whole available power will be used, i.e. $P_{t o t, 0}=P_{\max }$, and the power used by a streaming call is

$P_{d}^{s}=\frac{\alpha \beta^{s}}{1-\alpha \beta^{s}} P_{C C H}+\frac{\beta^{s}}{1-\alpha \beta^{s}}\left(\alpha P_{\max }+\chi P_{\max } \bar{F}+N_{0} \bar{q}\right)$.

For an HSDPA call, the achieved value of $\beta^{d}$ is equal to

$$
\beta^{d}=\frac{P_{d}^{d}}{\left(\alpha P_{\max }+\bar{\chi} P_{\max } \bar{F}_{i}+N_{0} \bar{q}_{i}\right)},
$$

where $P_{d}^{d}$ is the power received by a HSDPA call. Since a HSDPA user utilizes the whole available power while receiving, its power is:

$$
P_{d}^{d}=P_{\max }-P_{C C H}-P_{S C C H}-P_{d}^{s} X_{d}^{s}
$$

with $P_{S C C H}$ the power associated with the Shared Control Channel (SCCH), respectively. Therefore, the SINR of one HSDPA user can bne calculated using the definition of $\beta$ in Eqn. (11).

Knowing this SINR of HSDPA users, the choice of the modulation is based on link level curves $t(S I N R)$ as described in [14]. Thus, the overall HSDPA throughput in the cell is given by Eqn. (14) on the top of the next page, and the throughput of one HSDPA user is given by $\frac{T_{d}^{d}\left(X_{d}^{s}\right)}{X_{d}^{d}}$. Note that this throughput depends on the radio conditions of data users in the cell (through the mean path loss and F-factor).

Since we allow streaming calls in the network, the condition

$$
P_{\text {max }}-P_{C C H}-P_{S C C H}-P_{d}^{s} X_{d}^{s}>0
$$

has to be imposed in order to guarantee that there exists some power dedicated to this purpose. Thus, eqn. (15) provides the maximal number of UMTS calls

$$
N_{d}=\left\lfloor\frac{P_{\max }-P_{C C H}-P_{S C C H}}{\beta_{v}\left(\chi P_{\max } \bar{F}+N_{0} \bar{q}+\alpha P_{\max }\right)}\right\rfloor
$$

when there exists a nonzero number of HSDPA calls.

\section{B. UPLINK MODEL}

In the uplink, the $(S I N R)_{u}^{s}$ received from a streaming mobile of a given cell 0 must be greater than a given constant to guarantee the reception of the signal at the BS:

$$
(S I N R)_{u}^{s}=\frac{P_{u}^{s}}{I_{\text {intra }, 0}+I_{\text {inter }, 0}+N_{0}} \geq \tilde{\Delta}^{s}=\frac{E^{s}}{N_{0}} \frac{R_{u}^{s}}{W}
$$

$E^{s} / N_{0}$ is the minimum allowed ratio between the bit energy and the interference plus noise density, which guarantees the target quality of service in terms of bit error probability; $W / R_{u}^{s}$ is the processing gain, i.e., the ratio between the chip rate and the source bit rate, $N_{0}$ is the background noise, and $I_{\text {intra }, 0}$ and $I_{\text {inter }, 0}$ are the total powers received from other mobiles within the considered cell and all its neighbours.

$$
\begin{gathered}
I_{\text {intra }, 0}=X_{u}^{s} P_{u}^{s} \\
I_{\text {inter }, 0}=\sum_{j \neq 0} \sum_{i=1}^{X_{u, j}^{s}} P_{u}^{i, j}
\end{gathered}
$$

where $P_{u}^{i, j}$ is the power emitted by mobile $i$ in cell $j$. We introduce the factor $f=E\left[\sum_{j \neq 0} \sum_{i=1}^{X_{u, j}^{s}} \frac{q_{0}^{i, j}}{q_{j}^{i, j}}\right]$, where $q_{k}^{i, j}$ is the path loss between a user in cell $j$ and the base station of cell $k$. If $\bar{P}$ is the average received power by a base station of the system, and considering the minimal power that can achieve the target SIR, we obtain:

$$
\tilde{\Delta}^{s}=\frac{P_{u}^{s}}{X_{u}^{s} P_{u}^{s}+\bar{P} f+N_{0}-P_{u}^{s}}
$$

Defining

$$
\Delta^{s}=\frac{\tilde{\Delta}^{s}}{1+\tilde{\Delta}^{s}}
$$

we obtain:

$$
P_{u}^{s}=\Delta^{s}\left(X_{u}^{s} P_{u}^{s}+\bar{P} f+N_{0}\right)
$$

leading to:

$$
P_{u}^{s}=\frac{\left(\bar{P} f+N_{0}\right) \Delta^{s}}{1-X_{u}^{s} \Delta^{s}}
$$

To ensure a good functioning of the system, we must fix a constraint on the load of the cell. Note that this load is defined by:

$$
\chi=\frac{I_{t o t}}{I_{t o t}+N_{0}}
$$

where $I_{t o t}$ is the overall power received by the base station:

$$
I_{\text {tot }}=X_{u}^{s} P_{u}^{s}+\bar{P} f
$$

And the uplink constraint is

$$
X_{u}^{s} \Delta^{s} \leq \frac{\left(\bar{P} f+N_{0}\right) \chi_{\max }-\bar{P} f}{N_{0}}
$$

and the capacity of the system in the uploink is given by:

$$
C_{u}=\left\lfloor\frac{\left(\bar{P} f+N_{0}\right) \chi_{\max }-\bar{P} f}{N_{0} \Delta^{s}}\right\rfloor
$$

For data calls, and in the absence of HSUPA, they may be carried on Dedicated (DCH) or Common Packet CHannels 


$$
T_{d}^{d}\left(X_{d}^{s}\right)=t\left(\frac{P_{\max }-P_{C C H}-P_{S C C H}-\beta^{s}\left(\alpha P_{\max }+\chi P_{\max } \bar{F}+N_{0} \bar{q}\right) X_{d}^{s}}{\chi P_{\max } \bar{F}+N_{0} \bar{q}+\alpha\left(P_{C C H}+P_{S C C H}\right)+\alpha \beta_{v}\left(\alpha P_{\max }+\chi P_{\max } \bar{F}+N_{0} \bar{q}\right) X_{d}^{s}}\right)
$$

(CPCHs) [11]. DCHs are not suitable for these kinds of transmissions, as the code resources will be taken for the whole of the communication, $\mathrm{CPCH}$ is the most suitable channel. In the presence of $X_{u}^{s}$ streaming calls, the available power that can be accepted for the reception of $\mathrm{CPCH}$ is:

$$
P_{C P C H}\left(X_{u}^{s}\right)\left(=\frac{\chi_{\max } N_{0}}{1-\chi_{\max }^{U}}-\left(X_{u}^{s} P_{u}^{s}+\bar{P} f\right)\right.
$$

Using Eqn. (18), this leads to the value:

$$
\Delta_{C P C H}\left(X_{u}^{s}\right)=P_{C P C H}\left(X_{u}^{s}\right) \frac{1-X_{u}^{s} \Delta^{s}}{\left(\bar{P} f+N_{0}\right)}
$$

and using Eqn. (17), we obtain the throughput of data calls in the uplink:

$$
T_{u}^{d}\left(X_{u}^{s}\right)=\frac{W}{E^{d} / N_{0}} \frac{\Delta_{C P C H}\left(X_{u}^{s}\right)}{1-\Delta_{C P C H}\left(X_{u}^{s}\right)}
$$

where $E^{d} / N_{0}$ is the minimum allowed ratio between the bit energy and the interference plus noise density, required to decode a communication on the $\mathrm{CPCH}$ channel. 